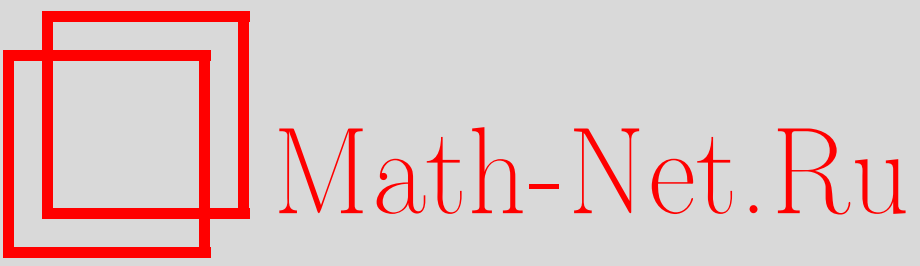

Ю. С. Вернов, М. Н. Мнацаканова, Регулярные представления обобщенной алгебры Гейзенберга, ТМФ, 2001, том 129, номер 2, 219-226

DOI: https://doi.org/10.4213/tmf531

Использование Общероссийского математического портала Math-Net.Ru подразумевает, что вы прочитали и согласны с пользовательским соглашением

http://www . mathnet.ru/rus/agreement

Параметры загрузки:

IP: 3.80 .181 .102

26 апреля 2023 г., $17: 45: 18$ 
ТЕОРЕТИЧЕСКАЯ

И МАТЕМАТИЧЕСКАЯ

ФИЗИКА

Том 129, № 2

ноябрь, 2001

(C) 2001 г.

Ю.С. Вернов*, М.Н. Мнацаканова ${ }^{\dagger}$

\section{РЕГУЛЯРНЫЕ ПРЕДСТАВЛЕНИЯ ОБОБЩЕННОЙ АЛГЕБРЫ ГЕЙЗЕНБЕРГА}

Исследованы общие свойства регулярных неприводимых представлений обобщенной алгебры Гейзенберга в произвольном невырожденном слабо замкнутом пространстве с индефинитной метрикой.

Алгебра Гейзенберга, иными словами алгебра канонических коммутационных соотношений (KKC), лежит в основе квантовой теории. Поэтому исследованию представлений этой алгебры посвящено множество работ, начиная с классических результатов Шредингера, Вейля, фон Неймана. Обзор основных результатов дан в работе [1], § 4. Большинство работ посвяшено изучению регулярных представлений алгебры Гейзенберга, а именно представлений, для которых KKC могут быть записаны как в стандартной, так и в вейлевской форме (при этом соответствуюшие группы должны быть непрерывны). Условие регулярности может быть сформулировано и непосредственно для обычных КKC - это условия Реллиха-Диксмье [2]. Согласно теореме фон Неймана каждое неприводимое регулярное представление алгебры Гейзенберга унитарно эквивалентно представлению Шредингера.

Напомним, что в этом представлении $\mathrm{KKC}\left[a, a^{+}\right]=1$ реализованы операторами

$$
a=\frac{1}{\sqrt{2}}\left(q+\frac{d}{d q}\right), \quad a^{+}=\frac{1}{\sqrt{2}}\left(q-\frac{d}{d q}\right)
$$

в пространстве $L_{2}(-\infty, \infty)$.

Ниже под алгеброй Гейзенберга мы будем понимать эту алгебру в случае одной степени свободы, поскольку случай произвольного, но конечного числа степеней свободы требует лишь очевидного обобщения.

В работах [3], [4] исследовались также более общие классы представлений КKC.

Как известно, ковариантное описание калибровочных полей требует введения пространства с индефинитной метрикой [5]-[7]. В работе [8] были найдены представления алгебры Гейзенберга в пространстве с индефинитной метрикой и сформулирован новый

\footnotetext{
${ }^{*}$ Институт ядерных исследований РАН, Москва, Россия. E-mail: vernov@ms2.inr.ac.ru

${ }^{\dagger}$ Научно-исследовательский институт ядерной физики Московского государственного университета, Москва, Россия. E-mail: mnatsak@theory.sinp.msu.ru
} 
критерий регулярности, эквивалентный условиям Реллиха-Диксмье в случае гильбертова пространства, но пригодный также и для пространства с индефинитной метрикой.

В последние годы широко изучались более обшие алгебры, чем алгебра Гейзенберга, а именно ее $q$ - и $R$-деформации (см. работы [9]-[14] и ссылки в них). Регулярные представления этих алгебр в пространстве с индефинитной метрикой были изучены в [15]-[17].

Как сама алгебра Гейзенберга, так и ее $q$ - и $R$-деформации являются частными случаями более общей алгебры, которую мы называем обобщенной алгеброй Гейзенберга. Эта алгебра была введена в [18], а в [16], [17] рассматривались некоторые ее свойства в связи с описанием представлений $q$ - и $R$-деформированных алгебр Гейзенберга.

Настоящая работа посвящена рассмотрению общих свойств обобщенной алгебры Гейзенберга в случае одной степени свободы.

Дадим определение рассматриваемой алгебры.

ОпреДЕлЕниЕ 1. Оператор $а$ и сопряженный ему относительно скалярного произведения $\langle\cdot, \cdot\rangle$, в общем случае индефинитного, оператор $a^{+}$порождают обобщенную алгебру Гейзенберга, если сушествует самосопряженный оператор $N$, удовлетворяющий условиям

$$
\begin{array}{cl}
N a=a(N-1), & N a^{+}=a^{+}(N+1), \\
a^{+} a=\varphi(N), & a a^{+}=\varphi_{1}(N),
\end{array}
$$

где $\varphi(N)$ и $\varphi_{1}(N)$ - некоторые функции.

Мы будем рассматривать представления этой алгебры в невырожденном замкнутом пространстве $J$, которое может быть как банаховым, так и пространством, замкнутым в слабом смысле. Последнее означает, что если при $n \rightarrow \infty$ скалярное произведение $\left\langle x_{n}, y\right\rangle$ имеет предел для любого $y \in J$, то сушествует элемент $x \in J$ такой, что $x=$ $\lim _{n \rightarrow \infty} x_{n}$.

Отметим, что если функции $\varphi(N)$ и $\varphi_{1}(N)$ таковы, что из условия (1) следует

$$
\varphi(N) a=a \varphi(N-1), \quad \varphi_{1}(N) a=a \varphi_{1}(N-1),
$$

TO

$$
\varphi_{1}(N)=\varphi(N+1)
$$

Действительно,

$$
a a^{+} a=\varphi_{1}(N) a=\varphi(N+1) a .
$$

В случае алгебры Гейзенберга $\varphi(N)=N$. Если представление рассматриваемой алгебры регулярно, то условие (3) всегда выполнено.

Подчеркнем, что в обшем случае операторы $a$ и $a^{+}$являются неограниченными. (Для алгебры Гейзенберга см. доказательство в работе [1], с. 2.) Поэтому обобшенная алгебра Гейзенберга определена не во всем пространстве $J$, а на некотором линейном множестве $D(A)$, плотном в слабом или сильном смысле, называемом областью определения $A$. Для простоты мы будем говорить, что $A$ определена в области $D(A)$.

Перейдем к определению регулярных представлений. Это можно сделать двумя эквивалентными способами. 
ОпреДЕлЕниЕ 2. Неприводимое представление обобщенной алгебры Гейзенберга регулярно, если оператор $N$ имеет собственный вектор

$$
N \psi_{\lambda}=\lambda \psi_{\lambda}
$$

$\psi_{\lambda}$ является циклическим вектором этого представления, которое строится следующим образом. Образуем цепочки векторов $\psi_{\lambda+n}=\left(a^{+}\right)^{n} \psi_{\lambda}$ и $\psi_{\lambda-n}=a^{n} \psi_{\lambda}$ (всюду $n, m \in \mathbb{N}, k, l \in \mathbb{Z})$. Согласно условию (1) каждый вектор из этих цепочек является собственным вектором оператора $N$ :

$$
N \psi_{\lambda+n}=(\lambda+n) \psi_{\lambda+n}, \quad N \psi_{\lambda-n}=(\lambda-n) \psi_{\lambda-n}
$$

Векторы $\psi_{\lambda+k}$ образуют полный набор собственных векторов оператора $N$, принадлежаших данному неприводимому представлению. Действительно,

$$
\begin{aligned}
a \psi_{\lambda+n} & =a a^{+} \psi_{\lambda+n-1}=\varphi(\lambda+n) \psi_{\lambda+n-1}, \\
a^{+} \psi_{\lambda-n} & =a^{+} a \psi_{\lambda-n+1}=\varphi(\lambda-n+1) \psi_{\lambda-n+1} .
\end{aligned}
$$

Следовательно, действие произвольного монома $M\left(a^{n}, a^{+m}\right)$, содержашего $n$ операторов $a$ и $m$ операторов $a^{+}$, на $\psi_{\lambda}$ пропорционально $\psi_{\lambda+m-n}$ :

$$
M\left(a^{n}, a^{+m}\right) \psi_{\lambda} \sim \psi_{\lambda+m-n}
$$

Далее, очевидно, что

$$
P_{n}\left(a, a^{+}\right) \psi_{\lambda}=\sum C_{k} \psi_{\lambda+k}
$$

где $P_{n}\left(a, a^{+}\right)$- произвольный полином от $a$ и $a^{+}$.

Некоторые шаги в исследовании обобщенной алгебры Гейзенберга могут быть сделаны независимо от конкретного вида функции $\varphi(N)$.

Построим сначала пространство $J$, исходя из пространства конечных линейных комбинаций $\psi_{\lambda+k}$, которое мы обозначим $J_{0}$. Очевидно, что $J_{0} \in D(A)$ для любой функции $\varphi(N)$.

Пространство $J$ замкнуто, следовательно, $J=\bar{J}_{0}$, однако замыкание $\bar{J}_{0}$ не является единственным, так как оно определяется топологией, заданной на $J_{0}$. Рассматриваемые представления во всех этих пространствах естественно считать эквивалентными, т.к. все они содержат плотную относительно слабой или сильной топологии область $J_{0}$ (см. обсуждение подобных проблем в работе [8]). Таким образом, мы можем ограничиться естественным замыканием, для построения которого используется исходное индефинитное скалярное произведение.

Подчеркнем, что $N$ не имеет нейтральных собственных векторов, т.к. $J$ и, следовательно, $J_{0}$ - невырожденные пространства. Итак, мы можем построить векторы

$$
e_{k}=\frac{\psi_{\lambda+k}}{\left|\left\langle\psi_{\lambda+k}, \psi_{\lambda+k}\right\rangle\right|^{\frac{1}{2}}},
$$


которые удовлетворяют условиям

$$
\left\langle e_{k}, e_{l}\right\rangle=\delta_{k l} \delta_{k}, \quad \delta_{k}= \pm 1
$$

Разделяя множество $e_{k}$ на множества положительных $e_{k}^{+}$и отрицательных $e_{k}^{-}$векторов, мы можем представить $J_{0}$ в виде

$$
J_{0}=J_{0}^{+} \oplus J_{0}^{-}, \quad J_{0}^{+} \perp J_{0}^{-} .
$$

Таким образом,

$$
x=x^{+}+x^{-}, \quad x^{ \pm}=\sum_{k} C_{k}^{ \pm} e_{k}^{ \pm}, \quad x^{ \pm} \in J_{0}^{ \pm} .
$$

Рассмотрим теперь пространство всех бесконечных линейных комбинаций $e_{k}$ таких, что $|\langle x, x\rangle|<\infty, \quad x=\sum_{k=0}^{\infty} C_{k} e_{k}$. Это пространство содержит все векторы $x^{ \pm}=$ $\sum_{k} C_{k}^{ \pm} e_{k}^{ \pm}$, удовлетворяющие условию

$$
\sum_{k=0}^{\infty}\left|C_{k}^{ \pm}\right|^{2}<\infty
$$

Условие (11) является достаточным для сушествования $\langle x, x\rangle$.

Но это условие является также необходимым, т.к. рассматриваемое пространство содержит векторы $x^{+}-x^{-}$, так же как и $x^{+}+x^{-}$, и, следовательно, оно содержит векторы $x^{ \pm}$. Таким образом, это естественное замыкание определяется как

$$
\bar{J}_{0}=\bar{J}_{0}^{+} \oplus \bar{J}_{0}^{-}
$$

Рассматриваемая алгебра $A$ определена в плотной области $D(A)$, конкретный вид которой зависит от свойств функции $\varphi(N)$.

Покажем, что если функция $\varphi(N)$ задана, то произвольное неприводимое регулярное представление полностью определяется спектром $N$. Это значит, что если $R$ и $R^{\prime}$ являются представлениями рассматриваемой алгебры и $\operatorname{Sp} N=\operatorname{Sp} N^{\prime}, \varphi(N)=$ $\varphi^{\prime}\left(N^{\prime}\right), \quad\left\langle\psi_{\lambda}, \psi_{\lambda}\right\rangle=\left\langle\psi_{\lambda}^{\prime}, \psi_{\lambda}^{\prime}\right\rangle$, то сушествует унитарный оператор $V$ такой, что $a^{\prime}=$ VaV $V^{+}$, $\left(a^{\prime}\right)^{+}=V a^{+} V^{+}$.

Достаточно показать, что все скалярные произведения $\left\langle\psi_{\lambda+k}, \psi_{\lambda+k}\right\rangle$ полностью определены функцией $\varphi(N)$, если $\lambda$ фиксирована.

Действительно, в соответствии с равенствами (7)

$$
\begin{aligned}
& \left\langle\psi_{\lambda+n}, \psi_{\lambda+n}\right\rangle=\left\langle\psi_{\lambda+n-1}, a a^{+} \psi_{\lambda+n-1}\right\rangle=\varphi(\lambda+n)\left\langle\psi_{\lambda+n-1}, \psi_{\lambda+n-1}\right\rangle \\
& \left\langle\psi_{\lambda-n}, \psi_{\lambda-n}\right\rangle=\left\langle\psi_{\lambda-n+1}, a^{+} a \psi_{\lambda-n+1}\right\rangle=\varphi(\lambda-n+1)\left\langle\psi_{\lambda-n+1}, \psi_{\lambda-n+1}\right\rangle .
\end{aligned}
$$

Из соотношений (13) и (14) непосредственно следует, что

$$
\begin{aligned}
\left\langle\psi_{\lambda+n}, \psi_{\lambda+n}\right\rangle & =\varphi(\lambda+n) \varphi(\lambda+n-1) \ldots \varphi(\lambda+1)\left\langle\psi_{\lambda}, \psi_{\lambda}\right\rangle, \\
\left\langle\psi_{\lambda-n}, \psi_{\lambda-n}\right\rangle & =\varphi(\lambda-n+1) \varphi(\lambda-n+2) \ldots \varphi(\lambda)\left\langle\psi_{\lambda}, \psi_{\lambda}\right\rangle .
\end{aligned}
$$


Так как $\left\langle\psi_{\lambda}, \psi_{\lambda}\right\rangle=\left\langle\psi_{\lambda}^{\prime}, \psi_{\lambda}^{\prime}\right\rangle$, то $\left\langle x^{\prime}, y^{\prime}\right\rangle=\langle x, y\rangle$, если

$$
\begin{gathered}
x=\sum_{k=l_{1}}^{l_{2}} C_{k} \psi_{\lambda+k}, \quad x^{\prime}=\sum_{k=l_{1}}^{l_{2}} C_{k} \psi_{\lambda+k}^{\prime}, \quad y=\sum_{k=m_{1}}^{m_{2}} \widetilde{C}_{k} \psi_{\lambda+k}, \quad y^{\prime}=\sum_{k=m_{1}}^{m_{2}} \widetilde{C}_{k} \psi_{\lambda+k}^{\prime}, \\
l_{1}, l_{2}, m_{1}, m_{2} \in \mathbb{Z} .
\end{gathered}
$$

Таким образом, мы доказали унитарную эквивалентность $R$ и $R^{\prime}$ в $J_{0}$ и $J_{0}^{\prime}$ и, следовательно, в $J$ и $J^{\prime}$.

Покажем теперь, что в произвольном регулярном представлении существует оператор $U(s) \equiv e^{i s N}, s \in \mathbb{R}$, обладаюший следуюшими свойствами:

$$
\begin{gathered}
U(s) U(t)=U(s+t), \\
U(s) \rightarrow \mathbb{I} \text { при } s \rightarrow 0, \quad U(0)=\mathbb{I}, \\
\lim _{s \rightarrow 0} \frac{U(s)-\mathbb{I}}{s} \psi=i N \psi, \quad \text { если } \psi \in D(N)
\end{gathered}
$$

(I - единичный оператор). Для того чтобы убедиться в этом, достаточно определить оператор $U(s)$ на векторах базиса:

$$
U(s) \psi_{\lambda+k}=e^{i s(\lambda+k)} \psi_{\lambda+k} .
$$

Первое условие в (17) проверяется непосредственно. Более того, легко видеть, что оператор $U(s)$ ограничен. Действительно,

$$
U(s) \sum C_{k} \psi_{\lambda+k}=\sum C_{k}^{\prime} \psi_{\lambda+k}, \quad C_{k}^{\prime}=e^{i s(\lambda+k)} C_{k} .
$$

Докажем последнее из свойств (17). Заметим, что для произвольного $\psi \in J_{0}$ наше утверждение сразу вытекает из равенства (18). Так как $U(s)$ - ограниченный оператор, утверждение остается справедливым всегда, когда существует вектор $N \psi$, т.е. если $\psi \in D(N)$. Из соотношения (18) следует, что

$$
U(2 \pi k)=e^{i 2 \pi k \lambda} .
$$

Легко проверить равенства

$$
U(s) a=e^{-i s} a U(s), \quad U(s) a^{+}=e^{i s} a^{+} U(s) .
$$

Следовательно,

$$
[U(2 \pi k), a]=\left[U(2 \pi k), a^{+}\right]=0 .
$$

Заметим, что можно найти собственный вектор $N$, действуя операторами $W_{k}(2 \pi)$ на произвольный вектор $\psi$, где

$$
W_{k}(s)=\int_{0}^{s} e^{-i k s^{\prime}} V\left(s^{\prime}\right) d s^{\prime}, \quad V(s)=e^{-i \lambda s} U(s) .
$$

Действительно,

$$
W_{k}(2 \pi) \psi=2 \pi C_{k} \psi_{\lambda+k}, \quad \psi=\sum_{l} C_{l} \psi_{\lambda+l} .
$$

Мы показали сушествование оператора $U(s)$ в произвольном регулярном представлении.

Докажем теперь, что и наоборот, существование оператора $U(s)$ означает, что у оператора $N$ есть собственньй вектор. Таким образом, мы можем дать следующее определение регулярного представления обобщенной алгебры Гейзенберга: 
ОПредЕлЕниЕ 3. Представление обобщенной алгебры Гейзенберга называется регулярным, если сушествует оператор $U(s)$, удовлетворяющий условиям (17).

ЗАмЕчАнИЕ 1. Достаточно предположить, что условия (17) выполнены в некоторой области $D$, инвариантной относительно действия операторов $U(s), s \in \mathbb{R}$.

Отметим сначала, что оператор $U(s)$ унитарен, т.к. оператор $N$ самосопряжен. Действительно, из условий (17) вытекает, что

$$
\frac{d}{d s} U(s)=i N U(s)
$$

следовательно,

$$
\frac{d}{d s}\langle U(s) \psi, U(s) \psi\rangle=0 \quad \forall \psi
$$

Далее, соотношения (20) по-прежнему справедливы, т.к. они вытекают из равенств

$$
\begin{aligned}
\frac{d}{d s}(U(s) a U(-s)) & =i U(s)[N, a] U(-s)=-i U(s) a U(-s), \\
\frac{d}{d s}\left(U(s) a^{+} U(-s)\right) & =i U(s)\left[N, a^{+}\right] U(-s)=i U(s) a^{+} U(-s) .
\end{aligned}
$$

Таким образом, для произвольного $k \in \mathbb{Z}$ оператор $U(2 \pi k)$ коммутирует с $a$ и $a^{+}$. Поэтому $U(2 \pi k)=C_{k} \mathbb{I}$ для неприводимых представлений. Детальное обсуждение этих вопросов можно найти в работе [8].

Так как $U(s)$ - унитарный оператор, то

$$
U(2 \pi)=e^{i 2 \pi \lambda}, \quad \lambda \in \mathbb{R} .
$$

Соотношение (26) эквивалентно (19).

Мы можем построить собственный вектор $N$, как и выше, с помошью оператора $W_{k}(2 \pi)$ (см. формулу (22)). Действительно, для любого $\psi \in D$ вектор $\psi_{k}=W_{k}(2 \pi) \psi$ либо является собственным вектором $V(s)$ при произвольном $s$, либо $\psi_{k}=0$ :

$$
V(s) \psi_{k}=\int_{0}^{2 \pi} e^{-i k s^{\prime}} V\left(s+s^{\prime}\right) \psi d s^{\prime}=e^{i k s} \int_{s}^{2 \pi+s} e^{-i k s^{\prime}} V\left(s^{\prime}\right) \psi d s^{\prime}=e^{i k s} \psi_{k}
$$

$(V(2 \pi)=\mathbb{I}$ в соответствии с $(26))$.

Для доказательства (4) осталось перейти к пределу $s \rightarrow 0$.

ЗАмЕчАнИЕ 2 . Существование оператора $W_{k}(s)$ обусловлено полнотой пространства $J$. Достаточно, чтобы $J$ было полным относительно слабой топологии [8]. Если $J-$ банахово пространство, то $W_{k}(s)$ сушествует, т.к.

$$
\left|V\left(s^{\prime}+\Delta\right)-V\left(s^{\prime}\right)\right|<\epsilon(\Delta)
$$

для любого $s^{\prime} \in(0, s)$. Действительно, в противном случае должна сушествовать последовательность $s_{n}, s_{n} \rightarrow \tilde{s}, n \rightarrow \infty, \tilde{s} \in(0, s)$, такая, что $V\left(s_{n}\right) \not t V(\tilde{s})$, а это противоречит непрерывности $V(s)$. 
ЗАмечаниЕ 3 . Для любого $\psi \in D$ существует $k_{0}$ такое, что $\psi_{k_{0}} \neq 0$. Этот вывод следует из невырожденности пространства $J$. Если для произвольного $k \in \mathbb{Z} \quad \psi_{k}=0$, то для любого вектора $\Phi$ выполняется соотношение

$$
\int_{0}^{2 \pi} e^{-i k s} T(s) d s=0
$$

где $T(s)=\langle\Phi, V(s) \Psi\rangle . T(s)$ - периодическая функция $(T(2 \pi)=T(0))$, все коэффициенты ряда Фурье которой равны нулю. Поэтому $T(s)=0$ для любого $s$ и $\Psi \perp \Phi$ для любого $\Phi$, откуда следует, что $\Psi=0$.

Отметим, что собственные векторы оператора $N$ могут принадлежать не множеству $D$, а только его замыканию $\bar{D}$. В этом случае $D$ - множество быстро сходяшихся бесконечных последовательностей $\sum C_{k} \psi_{\lambda+k}$. В случае алгебры Гейзенберга $C_{k}$ удовлетворяют условию: $C_{k} k^{n} \rightarrow 0, k \rightarrow \infty$ для любого $n$.

Имея $\psi_{\lambda}$, мы можем построить полный набор собственных векторов оператора $N$, соответствуюших неприводимому представлению, для которого $\psi_{\lambda}$ является циклическим вектором.

В заключение перечислим все виды регулярных неприводимых представлений рассматриваемой алгебры.

1. Спектр, ограниченный снизу. В этом случае сушествует такое число $n$, что $\varphi(\lambda-n)=0$ и, в соответствии с формулой $(16),\left\langle\psi_{\lambda-n-1}, \psi_{\lambda-n-1}\right\rangle=0$. Как мы уже отмечали, вследствие невырожденности $J$ у оператора $N$ нет нейтральных векторов и поэтому $\psi_{\lambda-n-1}=0$. Если $\operatorname{Sp} N$ не ограничен сверху, то

$$
\text { Sp } N=\lambda-n+m \text {. }
$$

Так как рассматриваемое представление неприводимо, каждый собственный вектор оператора $N$ можно считать циклическим. Из нашего рассмотрения непосредственно следует, что начиная с любого собственного вектора $N$, мы придем к тому же самому множеству собственных векторов $N$ и, следовательно, к тому же самому пространству $J_{0}$. Более того, мы можем начать с любого $x \in J_{0}$. Поэтому такое представление будет неприводимым по построению. В качестве циклического вектора удобно выбрать вектор $\psi_{\lambda-n}$. Мы можем определить такие представления как фоковские представления, так как

$$
\psi_{\lambda-n-1}=a \psi_{\lambda-n}=0 .
$$

2. Спектр, ограниченный сверху. В этом случае $\psi_{\lambda+m+1}=0$ для некоторого $m$, что означает

$$
a^{+} \psi_{\lambda+m}=0
$$

в согласии с соотношением (15). Естественно назвать этот случай антифоковским.

Можно считать $\psi_{\lambda+m}$ шиклическим вектором. Очевидно, что $\operatorname{Sp} N=\lambda+m-n$.

3. Конечный спектр. В этом случае соотношения (27) и (28) выполняются одновременно и $\operatorname{Sp} N=\lambda-n, \lambda-n+1, \ldots, \lambda+m$. Такого вида $\operatorname{Sp} N$ не сушествует в алгебре Гейзенберга, но он возможен в ее $q$ - и $R$-деформациях.

3 Теоретическая и математическая физика, т. 129, № 2, 2001 г. 
4. Неограниченный спектр. В этом случае $\varphi(\lambda+k) \neq 0$ для любого $k$, следовательно, $a \psi_{\lambda+k} \neq 0, a^{+} \psi_{\lambda+k} \neq 0$.

Итак, мы видим, что рассмотрение определенной обобшенной алгебры Гейзенберга сводится к изучению свойств соответствуюшей функции $\varphi(\lambda+k)$.

\section{Список литературы}

[1] C. R. Putnam. Commutation Properties of Hilbert Space Operators and Related Topics. Berlin-Heidelberg-New York: Springer, 1967.

[2] F. Rellich. Nach. Akad. Wiss. Gött. Math. -Phys. Klasse. 1946. P. 107; J. Dixmier. Comp. Math. 1958. V. 13. P. 263.

[3] F. Acerbi, G. Morchio, F. Strocchi. Infrared singular fields and non-regular representations of CCR algebras. Preprint SISSA 39/92/FM, 1992; Lett. Math. Phys. 1993. V. 27. P. 1.

[4] K. Schmüdgen. J. Funct. Analysis. 1983. V. 50. P. 8; Publ. RIMS. Kyoto Univ. 1983. V. 19. P. 601.

[5] F. Strocchi and A. S. Wightman. J. Math. Phys. 1974. V. 15. P. 2198.

[6] G. Morchio and F. Strocchi. Ann. Inst. Henri Poincaré. 1980. V. 33. P. 251.

[7] F. Strocchi. Selected Topics on the General Properties of Quantum Field Theory. Singapore: World Scientific, 1993.

[8] M. Mnatsakanova, G. Morchio, F. Strocchi, and Yu. Vernov. J. Math. Phys. 1998. V. 29. P. 2969.

[9] L. D. Faddeev. Integrable models in $(1+1)$-dimensional quantum field theory. In: Recent Advances in Field Theory and Statistical Mechanics. Les Houches, session XXXIX. Eds. J.-B. Zuber and R. Stora. Amsterdam: Elsevier, 1984. P. 563.

[10] P. P. Kulish and E. K. Sklyanin. Quantum spectral transform method. Recent developments. In: Integrable Quantum Field Theory. Proc. of the Symp. held at Tvaerminne, Finland, 23-27 March, 1981. Lect. Notes Phys. V. 151. Eds. J. Hietarinta and C. Montonen. Berlin: Springer, 1982. P. 61.

[11] M. Chaichian and P. P. Kulish. Phys. Lett. B. 1990. V. 234. P. 72.

[12] P. P. Kulish and E. V. Damaskinsky. J. Phys. A. 1990. V. 23. Р. L415; П. П. Кулии. TMФ. 1991. T. 86. C. 8.

[13] M. S. Plyushchay. Nucl. Phys. B. 1997. V. 491. P. 619.

[14] M. S. Plyushchay. Mod. Phys. Lett. A. 1996. V. 11. P. 2953.

[15] M. Chaichian, M. N. Mnatsakanova, and Yu. S. Vernov. Analogue of the von Neumann's theorem of the q-oscillator algebra. In: Proc. of the XV Workshop "Problems on High Energy Physics and Field Theory", Protvino, 1992. Eds. A. P. Samokhin and G. L. Rcheulishvili. Protvino: IHEP, 1995. P. 126.

[16] Ю. С. Вернов, М. Н. Мначаканова. ТМФ. 1997. Т. 113. С. 355.

[17] Ю. С. Вернов, М. Н. Мнацаканова. ТМФ. 2000. Т. 125. С. 272.

[18] M. N. Mnatsakanova, G. Morchio, and Yu. S. Vernov. Canonical commutation relations in a positive and an indefinite metric spaces. In: Quarks-96 (Proc. 9th Int. seminar, Yaroslavl, Russia, May 5-11, 1996). V. 2. Eds. V. A. Matveev, A. A. Penin, V. A. Rubakov, and A. N. Tavkhelidze. Moscow: Inst. Nucl. Res., Russ. Acad. Sci, 1997. P. 51. 